\title{
Femtosecond Photodesorption of Small Molecules from Surfaces: A Theoretical Investigation from First Principles
}

\author{
Christiane P. Koch, ${ }^{*}$ Thorsten Klüner, ${ }^{\dagger}$ and Hans-Joachim Freund \\ Fritz-Haber-Institut der Max-Planck-Gesellschaft, Faradayweg 4-6, 14195 Berlin, Germany
}

Ronnie Kosloff

Fritz. Haber Research Center and Department of Physical Chemistry, The Hebrew University, Jerusalem 91904, Israel

(Received 22 October 2002; published 17 March 2003)

\begin{abstract}
A microscopic model for the excitation and relaxation processes in photochemistry at surfaces is developed. Our study is based on ab initio calculations and the surrogate Hamiltonian method treating surface electron-hole pairs as a bath of two-level systems. Desorption probabilities and velocities in the experimentally observed range are obtained. The excited state lifetime is calculated, and a dependence of observables on pulse length is predicted.
\end{abstract}

DOI: $10.1103 /$ PhysRevLett.90.117601

PACS numbers: 79.20.La, 68.43.Tj

Surface photochemistry occurs in many instances, including photocatalysis, atmospheric chemistry, and photochemical reactions at interstellar dust particles. Despite its ubiquitous nature, a microscropic understanding of the underlying basic processes remains a great challenge. In order to obtain a detailed mechanistic picture, the complexity of the studied systems and processes has to be reduced significantly. The simplest photochemical phenomenon on surfaces is laser-induced desorption of small molecules. Two-state models such as the Menzel-GomerRedhead or Antoniewicz scenarios [1] have proven useful to qualitatively understand the mechanism of DIET (desorption induced by electronic transitions). In both models, the excitation from the electronic ground to an excited state and the relaxation back to the ground state are modeled as vertical transitions. While significant progress has recently been made concerning reliable potential energy surfaces for DIET dynamics [2,3], excitation and relaxation mechanisms have been modeled only semiphenomenologically $[4,5]$ assuming a separation of time scales of excitation, excited state nuclear dynamics, and quenching. Since electronic relaxation occurs on the time scale of femtoseconds [5], this assumption breaks down with the introduction of femtosecond laser pulses.

Electronic relaxation is caused by the interaction of the excited adsorbate-substrate complex with electron-hole pairs in the surface [5]. In this Letter, we develop a microscopic model for this interaction assuming that electron-hole pairs can be described as a bath of twolevel systems (TLS). The surrogate Hamiltonian approach [6] is employed in which for a finite time the infinitely many TLS are approximated by a finite number. The finite time interval for which converged results can be obtained depends on the interaction strength and the number of TLS. This means that convergence is controllable and no further assumptions are needed. The surrogate Hamiltonian approach represents one possibility to treat a quantum system interacting with its environment.
Since it does not rely on the approximation of weak coupling between system and environment and a separation of time scales of system dynamics and relaxation, it is particularly well suited to describe ultrafast charge transfer events in condensed phase [7]. In the present study, the construction of the bath is tailored to model electron-hole pairs in insulator and semiconductor surfaces. A particularly well studied example where quenching by electron-hole pairs in an insulator surface is crucial is given by the laser-induced desorption of $\mathrm{NO}$ from $\mathrm{NiO}(100)$. We have therefore combined information from previous experimental $[8,9]$ and theoretical $[2,10]$ studies with the surrogate Hamiltonian method to develop a model which treats all steps of the DIET dynamics on the same level of rigor. The method is, however, of general applicability and has been employed to metal surfaces [6] and chromophores in solution [7] before.

We model the adsorbate on a finite part of the surface as primary system $\hat{\mathbf{H}}_{S}$, while electron-hole pairs in the surface are treated as environment or bath $\hat{\mathbf{H}}_{B}$. The effect of this environment on the primary system is captured in an interaction term $\hat{\mathbf{H}}_{S B}$ leading to electronic quenching. The total Hamiltonian can then be written as

$$
\hat{\mathbf{H}}_{\mathrm{tot}}=\hat{\mathbf{H}}_{S}+\hat{\mathbf{H}}_{S F}(t)+\hat{\mathbf{H}}_{S B}+\hat{\mathbf{H}}_{B},
$$

where the interaction of the system with a time-dependent laser field $\hat{\mathbf{H}}_{S F}(t)$ has been included.

The Hamiltonian of the primary system $\hat{\mathbf{H}}_{S}$ describes the ground state of the NO molecule adsorbed on the surface and the negatively charged excited state,

$$
\hat{\mathbf{H}}_{S}+\hat{\mathbf{H}}_{S F}(t)=\left[\begin{array}{cc}
\hat{\mathbf{T}}+V_{g}(\hat{\mathbf{Z}}) & E^{*}(t) \hat{\mu}_{t r}(\hat{\mathbf{Z}}) \\
E^{*}(t) \hat{\mu}_{t r}(\hat{\mathbf{Z}}) & \hat{\mathbf{T}}+V_{e}(\hat{\mathbf{Z}})
\end{array}\right]
$$

$\hat{\mathbf{T}}$ is the (nuclear) kinetic energy operator which is applied in momentum space. $V_{g}$ is the ground state empirical potential, and $V_{e}$ the excited state $a b$ initio potential obtained within a valence configuration interaction (CI) 
framework [10]. The potentials have been constructed in 2 degrees of freedom-distance of the center of mass of the molecule from the surface $\hat{\mathbf{Z}}$ and the angle $\theta$ between the NO molecular axis and the surface normal. We restrict the treatment in the following to 1 degree of freedom, $\hat{\mathbf{Z}}$, and keep the polar angle fixed at its equilibrium value, $\theta=45^{\circ}$. The intramolecular distance of NO is kept constant ( $R=2.175$ a.u.). The interaction of the system with the laser field $E(t)$ induces an electronic transition from the surface to the NO molecule, i.e., the excitation is direct within the substrate-adsorbate complex. $\hat{\mu}_{t r}(\hat{\mathbf{Z}})$ is the transition dipole operator which is obtained from the oscillator strength known from CI calculations [10]. The field $E(t)$ is treated semiclassically, with a Gaussian pulse shape, $E(t)=E_{0} \exp \{-[(t-$ $\left.\left.\left.t_{\max }\right)^{2}\right] / 2 \sigma_{P}^{2}\right\} \exp \left(i \omega_{L} t\right)$. The standard deviation $\sigma_{P}$ is related to the full width at half maximum (FWHM) $\tau_{P}$ of the pulse $\left(\tau_{P}=2 \sigma_{P} \sqrt{2 \ln 2}\right)$.

The direct optical excitation as modeled in Eq. (2) is justified by experiments which found a dependence of the desorption yield on the polarization of the laser light [11]. This dependence indicates that optical selection rules and hence the symmetry of the involved states are important. We can furthermore assume a resonant transition since many excited states are located in the energy range probed by the laser pulse [2]. This argument is supported by the experimental finding that state resolved velocity distributions are independent of the laser energy $\hbar \omega_{L}$ [8].

The electron-hole pairs, i.e., the $\mathrm{O} 2 p \rightarrow \mathrm{Ni} 3 d$ charge transfer states, are described as a bath of TLS,

$$
\hat{\mathbf{H}}_{B}=\varepsilon \sum_{i} \hat{\sigma}_{i}^{+} \hat{\boldsymbol{\sigma}}_{i}+\frac{\eta}{\log (N)} \sum_{i j(N N)}\left(\hat{\sigma}_{i}^{+} \hat{\boldsymbol{\sigma}}_{j}+\hat{\boldsymbol{\sigma}}_{j}^{+} \hat{\boldsymbol{\sigma}}_{i}\right),
$$

where $(N N)$ stands for nearest neighbor. The $i$ th TLS models the electron-hole pair located at site $i$, where the sites are the Ni-O pairs in the lattice. The spin matrix $\hat{\sigma}_{i}^{+}$ $\left(\hat{\sigma}_{i}\right)$ creates (annihilates) the TLS at site $i$. Equation (3) represents an extremely simple model of the electronic structure of nickel oxide. It is, however, sufficient for the present purpose of studying the influence of the surface on the desorption dynamics. Connecting Eq. (3) to $a b$ initio electronic structure calculations enables us to capture the main features. The first term of Eq. (3) describes the excitation of localized electron-hole pairs/ TLS. This is motivated by the generally localized nature of the Ni $3 d$ states. Delocalization is brought about by the $\mathrm{O} 2 p$ states and introduced into the model by the second term in Eq. (3). This term describes the transport of excitation from one electron-hole pair to its nearest neighbors. Two quantities characterize the bath: the excitation energy $\varepsilon$ and the nearest neighbor interaction strength $\eta$. The latter quantity leads to a finite width of excitation energy, i.e., an energy "band" of the bath: If the bath Hamiltonian, Eq. (3), is diagonalized, and $N$ is the number of modes, one obtains $N$ energies around $\varepsilon$ corresponding to single excitations, $N$ energies around $2 \varepsilon$ corresponding to double excitations, etc. The spread of these eigenvalues around $\varepsilon$ is determined by $\eta$. $\varepsilon$ can be viewed as the center of the bath energy band and is therefore related to the band gap. The scaling $1 / \log (N)$ results from the topology of the problem, i.e., from the mapping of the two horizontal dimensions of the bath onto one dimension [12].

Since the TLS bath represents the electronic structure properties of $\mathrm{NiO}, \varepsilon$ and $\eta$ should be chosen such that the eigenvalues of the bath display the band gap and the width of excitations correctly. However, in order to gain further insight into our model, we can vary these quantities freely to study their influence on the dynamics. We found that only a value of $\varepsilon$ which gives the correct band gap leads to exponential decay of population of the excited state. $\eta$, however, determines the convergence properties of the model. The width of existing single electronic excitations in $\mathrm{NiO}$ is about $10 \mathrm{eV}$ [12]. However, the laser pulse probes only a small window of this band. Therefore $\eta$ was chosen to give the best possible convergence. We have verified that other choices of $\eta$ give similar results requiring, however, a larger number of bath modes [12]. Double excitations are expected to be negligible for the relaxation dynamics because their energies are also much higher than the energy window probed by the pulse (energies between 5.5 and $10 \mathrm{eV}$ as opposed to energies up to about $4 \mathrm{eV}$ ). This has been confirmed in the simulations. The values of $\varepsilon$ and $\eta$ in the calculations presented below are $\varepsilon=3.7 \mathrm{eV}$ and $\eta=0.7 \mathrm{eV}$. Furthermore, the surface was split into layers of Ni-O pairs, and each layer was treated as a separate bath. Then in addition to the nearest neighbor interaction between electron-hole pairs within one surface layer [ $\eta$ of Eq. (3)], a coupling between the layers, $\eta_{L}$, is introduced. While $\eta$ describes the transport of excitation horizontally, $\eta_{L}$ causes transport of excitation into the surface. The values of the two quantities should therefore be similar. The two processes although equally likely for nickel oxide are not completely treated on the same footing in the model. This separation is possible due to the electronic structure of nickel oxide, in particular, due to the localized $d$ orbitals. The details of these investigations as well as of the bath mapping will be reported elsewhere [12].

The interaction of electron-hole pairs with the $\mathrm{NO}^{-}$-like intermediate causes a quenching of electronic excitation of the primary system,

$$
\hat{\mathbf{H}}_{S B}=\left(\begin{array}{ll}
0 & 1 \\
1 & 0
\end{array}\right) \otimes \sum_{i} \hat{\mathbf{V}}_{i}\left(\hat{\sigma}_{i}^{+}+\hat{\sigma}_{i}\right) .
$$

Since electron-hole pairs represent dipoles, and the laser excitation creates a nonzero transition dipole $\hat{\vec{\mu}}_{S}$ in the system, the interaction is modeled as dipole-dipole interaction, $\hat{\mathbf{V}}_{i}=\hat{\vec{\mu}}_{S} \cdot \vec{E}_{i}$. This assumes that the electric field of the bath dipoles $\vec{E}_{i}$ can be described classically and 
that the system dipole is in the far field region. These additional approximations are expected to be negligible compared to the simplification of the $\mathrm{O} 2 p \rightarrow \mathrm{Ni} 3 d$ charge transfer states to TLS. The electric field of the $i$ th bath dipole is determined by the dipole strength $\left|\hat{\vec{\mu}}_{i}\right|=q a_{0} / 2$ and the distance of the $i$ th site from the NO molecule. $a_{0}$ denotes the lattice constant of $\mathrm{NiO}$ $\left(a_{0}=3.93 \AA\right)$. The only quantity which enters the interaction Hamiltonian, Eq. (4), is the dipole charge $q$. It characterizes the completeness of charge transfer between the oxygen and nickel ions. An estimate for the value of $q$ is known from a population analysis of the $\mathrm{O} 2 p \rightarrow \mathrm{Ni} 3 d$ charge transfer states obtained in CI calculations, $0.1 \leqq q \leqq 0.2[10]$.

The simulations start with the vibrational ground state of the electronic ground state potential. Because of the large band gap, the bath is not excited thermally at time $t=0$. The time evolution of the wave function was calculated using the Chebychev propagator [13]. The interaction between primary system and bath was switched off when recurrences were observed. The ground state wave packet was then propagated until norm and shape of the wave packet in the asymptotic region of the potential $\left(V_{g} \approx 0\right)$ were converged.

Figure 1 (left) presents the excited state population vs time in a linear and semilogarithmic scale for different values of the dipole charge $q$. The envelope of the pulse $\left(\tau_{P}=5 \mathrm{fs}\right)$ is indicated. The excited state population is plotted until recurrences occur, i.e., the length of the curves indicates convergence. The results for two different numbers of bath modes, $N=61$ and $N=63$, are compared. Exponential decay is observed after the excitation of population due to the pulse. Increasing the dipole charge $q$ leads to a stronger interaction between system and bath and therefore to a smaller lifetime of the excited state. Furthermore, the maximum population of the excited state is decreased for larger $q$. For large $q$, no significant population of the excited state is observed at all. This corresponds to the case of metals where a direct

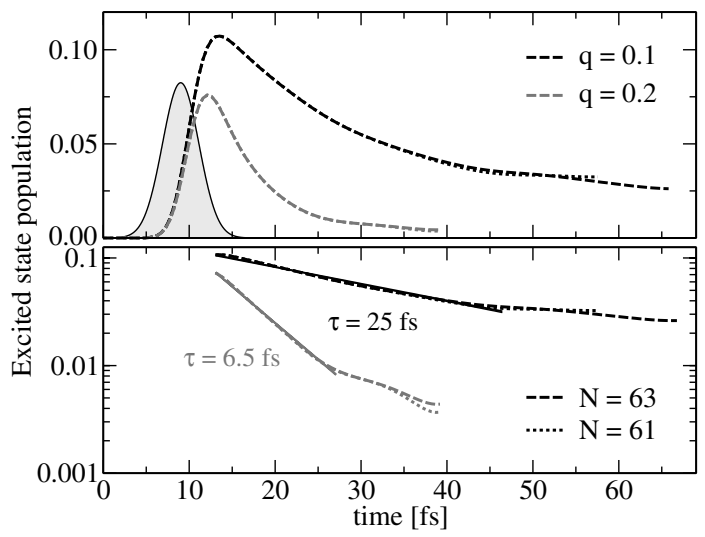

FIG. 1. Dependence on the dipole charge $q$ characterizing the system-bath interaction strength. optical excitation is immediately quenched due to the strong interaction with the substrate. Decay rates or lifetimes, respectively, were obtained from fitting the data to an exponential. The lifetime of the electronic state has previously been estimated as about 25 fs [2]. With the surrogate Hamiltonian, such a lifetime is obtained for a value of $q \approx 0.1$. This value of $q$ agrees very well with the estimate from the CI calculations. In contrast to the semiempirical guess [2], we can now calculate the excited state lifetime on an $a b$ initio basis.

The lifetime of the electronically excited state and therefore $q$ determine the desorption probability. The desorption probability is obtained by weighting the norm in the asymptotic region by the excitation probability. It is shown for $q=0.1$ in Fig. 2. Desorption probabilities between $1 \%$ and $20 \%$ were obtained. This is compatible with estimates from experiment [9]. The desorption velocities (not displayed, but cf. Fig. 3) are found to be in the experimentally observed range between 0 and $2000 \mathrm{~m} / \mathrm{s}$ [8,9]. Up to 21 layers with $N \leq 51$ and up to 13 layers with $N \leq 101$ bath modes in each layer were considered. The maximum convergence time was about 90 fs. This was sufficiently long to obtain converged desorption probabilities and velocities (cf. Fig. 2) within an error of at most $5 \%$. This error results from neglecting the remaining population of the excited state when the interaction between system and bath is switched off. The desorption probability in Fig. 2 is plotted for different values of the quantity $\eta_{L}$ which characterizes the coupling between different layers of $\mathrm{Ni}-\mathrm{O}$ pairs.

So far we have shown that the quantities of our model can be connected to ab initio calculations and that the obtained desorption probabilities and velocities are compatible with experimental results. The control parameter which is most directly accessible in experiment is the shape of the laser pulse, in particular, its intensity and duration. Figure 3 presents velocity distributions for increasing pulse duration with laser fluence kept constant. More than one peak can be observed in the velocity distributions. The appearance of additional peaks is



FIG. 2. Converged desorption probabilities. The results are well within the experimentally observed range. 


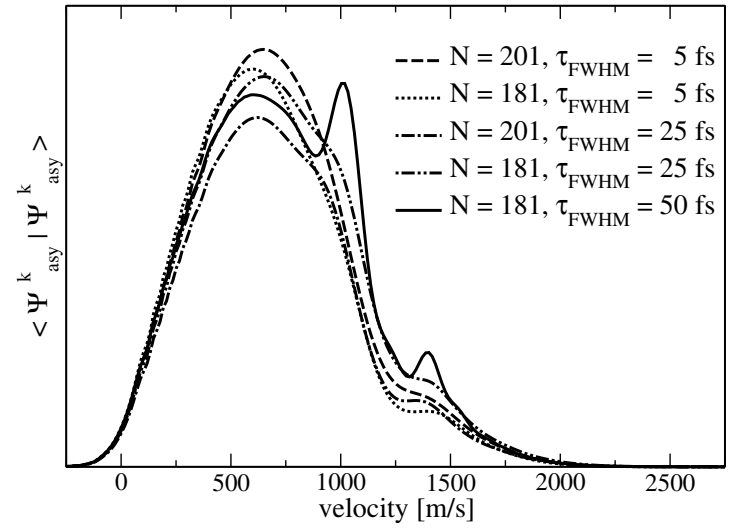

FIG. 3. Dependence of the shape of final velocity distributions on pulse duration.

related to the excited state wave packet passing the inner classical turning point. This requires the quenching to be moderate enough so that population reaches the classical turning point. The structure of the velocity distribution can thus be explained by quantum interferences due to different pathways of partial wave packets which undergo electronic quenching at different times. Figure 3 shows the interplay between pulse and nuclear dynamics once the pulse duration becomes comparable with the vibrational period of the excited state potential with respect to the molecule-surface bond. A longer pulse duration excites partial wave packets at times further away from each other. This leads to more different pathways becoming visible in the velocity distributions. While this is consistent within the model, some caution is advisable when drawing conclusions with respect to experiment. Quantum coherences will be attenuated in a higher dimensional description. They will furthermore be washed out by electronic dephasing which has been neglected in the present treatment.

The results of Fig. 3 suggest that the experimentally observed bimodality can be explained by quantum interferences due to different pathways. An alternative explanation was suggested within a previous two-dimensional stochastic wave packet treatment considering the center of mass distance from the surface $Z$ and the polar angle $\theta$ [2]. The bimodality was then connected to a bifurcation of the wave packet on the excited state which is caused by the topology of the excited state potential energy surface. A two-dimensional study with the surrogate Hamiltonian would allow for wave packet bifurcation as well as the passage of the classical turning point as cause of the quantum interferences. The extension of the present study to two dimensions is therefore the logical next step. The two hypotheses could then be tested by theoretical studies (but also by an experiment) which change the vibrational frequencies of the potential while leaving the chemistry invariant. This could be accomplished, for example, by using different isotopes of NO.

In conclusion, we presented the first microscopic model for electronic relaxation in laser-induced desorption. All quantities of the model were connected to estimates from $a b$ initio calculations. Desorption probabilities and velocities in the experimentally observed range were obtained without further assumptions. Our results suggest a dependence of velocity distributions on pulse duration once the pulse FWHM is comparable to the vibrational period of the excited state potential. To the best of our knowledge, our approach is the first which treats every step of the DIET process on the same level of rigor. In particular, it allowed us to calculate the excited state lifetime from first principles. This result is crucial for the design of future experiments on coherent control of surface processes or two pulse studies such as two photon photoemission (2PPE) where the length of the pulses must compete with the excited state lifetime. The bimodality of experimentally observed velocity distributions, in particular, a possible control of their shape, needs to be addressed by further theoretical as well as experimental studies.

We wish to thank Roi Baer for fruitful discussions. Financial support from the German-Israeli Foundation (GIF) and from the Deutsche Forschungsgemeinschaft (SPP 1093) is gratefully acknowledged. The Fritz Haber Center is supported by the Minerva Gesellschaft für die Forschung GmbH München, Germany.

*Email address: koch_c@fhi-berlin.mpg.de

${ }^{\dagger}$ Email address: kluener@fhi-berlin.mpg.de

[1] D. Menzel and R. Gomer, J. Chem. Phys. 41, 3311 (1964); P. A. Redhead, Can. J. Phys. 42, 886 (1964); P. R. Antoniewicz, Phys. Rev. B 21, 3811 (1980).

[2] T. Klüner et al., Phys. Rev. Lett. 80, 5208 (1998); T. Klüner et al., in Laser Techniques for Surface Science III, SPIE Proceedings Vol. 3272 (SPIE-International Society for Optical Engineering, Bellingham, WA, 1998), p. 177.

[3] S. Thiel et al., Phys. Rev. Lett. 87, 077601 (2001).

[4] J. Gadzuk, Surf. Sci. 342, 345 (1995).

[5] H. Guo et al., Prog. Surf. Sci. 62, 239 (1999).

[6] R. Baer and R. Kosloff, J. Chem. Phys. 106, 8862 (1997).

[7] C. P. Koch et al., J. Chem. Phys. 116, 7983 (2002).

[8] G. Eichhorn et al., J. Chem. Phys. 111, 386 (1999).

[9] T. Mull et al., J. Chem. Phys. 96, 7108 (1992).

[10] T. Klüner et al., J. Chem. Phys. 104, 10030 (1996); T. Klüner et al., J. Mol. Catal. A 119, 155 (1997).

[11] H. Zacharias et al., Appl. Phys. B 68, 605 (1999).

[12] C. P. Koch, T. Klüner, H.-J. Freund, and R. Kosloff (to be published).

[13] H. Tal-Ezer and R. Kosloff, J. Chem. Phys. 81, 3967 (1984). 